\title{
El nuevo Fondo Europeo de Apoyo a la Paz. Un análisis dentro de la PCSD
}

\section{The new European Peace Facility. An analysis within the CSDP}

\section{Miguel EIROS BACHILLER}

Universidad de Salamanca

meiros@usal.es

ORCID: https://orcid.org/0000-0002-4691-3521

\author{
(c) () () Este artículo está sujeto a una licencia “Creative Commons Reconocimiento- \\ No Comercial” (CC-BY-NC). \\ DOI: https://doi.org/ 10.24197/ree.79.2022.139-162
}

Resumen: El nuevo Fondo Europeo de Apoyo a la Paz fue aprobado el pasado 22 de marzo de 2021. Este mecanismo supone la sustitución de dos instrumentos anteriores; el mecanismo ATHENA y el African Peace Facility. Todavía es pronto para ver las consecuencias, pero está claro que este nuevo mecanismo le dará a la UE mayor autonomía y eficacia a la hora de desplegar misiones militares y apoyar a terceros en el ámbito militar. Sin embargo, este nuevo Fondo tiene algunos inconvenientes que conviene ponderar. Por lo tanto, esta nueva herramienta supone un tímido avance en la PCSD, aunque con posibilidades de desplegar un mayor rango de acción. Todo depende de la voluntad de los participantes de este nuevo Fondo.

Palabras clave: Fondo Europeo de Apoyo a la Paz; mecanismo ATHENA; African Peace Facility; misiones militares de la UE; medidas de asistencia de la UE.

Abstract: The new European Peace Facility was approved on March 22, 2021. This mechanism replaces two previous instruments: the ATHENA mechanism and the African Peace Facility. It is still early to see the consequences, but this new mechanism will give the EU greater autonomy and efficiency in deploying military missions and supporting third parties in the military field. However, this new Fund has some drawbacks that should be considered. Therefore, this new tool represents a timid advance in the CSDP, although with the possibility of deploying a wider range of action. Everything depends on the will of the participants in this new Fund.

Keywords: European Peace Facility; ATHENA mechanism; African Peace Facility; EU military missions; EU assistance measures. 


\section{INTRODUCCIÓN}

A la hora de hablar de la financiación de las misiones militares, se puede comprobar que la cuestión que subyace de fondo es la falta de voluntad política y la ausencia de un proyecto común en materia de defensa. Se puede decir que la Unión Europea está aún a "años luz” de los objetivos del artículo 42.2 del Tratado de la Unión ${ }^{1}$.

Sin embargo, a pesar de todas estas contrariedades, esta materia ha ido avanzando con un paso lento pero seguro y siempre marcado por las circunstancias que le ha rodeado. Uno de estos momentos fue a partir de 2016 en el que la UE ha ido aprobando una serie de instrumentos para fortalecer la PCSD ${ }^{2}$ cuyo último elemento en aprobarse ha sido el nuevo Fondo Europeo de Apoyo a la Paz (FEAP) el pasado 22 de marzo de $2021^{3}$. Este nuevo mecanismo financiero cubrirá todas las acciones exteriores de la UE que tengan repercusiones en el ámbito militar o de la defensa. Tiene como objetivo último reforzar la capacidad de la UE para prevenir conflictos, mantener la paz y fortalecer la estabilidad y la seguridad internacional.

Este nuevo fondo viene a cubrir ciertas lagunas que estaban todavía vigentes debido a las carencias que presentaban los instrumentos anteriores. Por ejemplo, era llamativo la escasa cantidad que suponían los gastos del fondo en relación con los gastos que suponían las operaciones militares en su conjunto. También este nuevo fondo ampliará el concepto de gastos comunes y el alcance geográfico a escala mundial a la hora de prestar apoyos a otras entidades. Antes sólo se prestaba esa ayuda de la UE en operaciones de apoyo a la paz con liderazgo africano.

El nuevo FEAP supone un avance en determinadas cuestiones. Sin embargo, el objetivo último de este estudio es responder a la pregunta si las lagunas que viene a cubrir este Fondo presentan más ventajas que desventajas ya que han surgido voces críticas respecto a ciertos aspectos.

\footnotetext{
${ }^{1}$ Moreno Zamora, Juan Ignacio (2016), "La cuestión militar en la Unión Europea”, Instituto Español de Estudios Estratégicos, N. 3, p. 451.

${ }^{2}$ Para profundizar en esta reforma institucional en materia de defensa desde el 2016 vid. Besch, Sophia (2020), “EU’s Institutional Framework regarding Defence Matters”, Policy Department, Directorate-General for External Policies, Parlamento Europeo, Bruselas.

${ }^{3}$ Decisión (PESC) 2021/509 del Consejo de 22 de marzo de 2021 por la que se crea un Fondo Europeo de Apoyo a la Paz y se deroga la Decisión (PESC) 2015/528. DO L 102 de 24.3.2021.
} 
En definitiva, se trata de comprobar, si realmente el nuevo FEAP puede utilizarse como una herramienta que vaya más allá, y pueda ser considerado como un mecanismo clave en el proceso de integración en materia de la PCSD.

\section{EL SISTEMA DE FINANCIACIÓN DE EN EL ÁMBITO MILITAR Y DEFENSA}

\section{1. De Maastricht hasta Lisboa}

La Política de defensa ha sido siempre un tema delicado y de difícil consenso en la UE. Después del AUE, algunos autores han calificado de "esquizofrenia" la división existente en aspectos de seguridad y defensa ${ }^{4}$. Otros, prefieren subrayar que el problema radica en mismo concepto de seguridad europea.

En la década de los noventa, el factor determinante de aceleración del proceso de integración no se encuentra tanto en el marco jurídico, sin duda importante, sino en la crisis de Kosovo de 1998. Este hecho movilizó la voluntad política de los Estados miembros que hasta entonces era nula en el avance de la PESD ${ }^{5}$.

Hasta entonces son bien conocidas las reticencias de la mayoría de los Estados miembros de la UE a comprometerse de forma directa en las labores del mantenimiento de la paz mediante el envío de unidades militares dirigidas por otros organismos. En esta década fueron las experiencias traumáticas de las tropas enviadas a países como Somalia, Bosnia y Ruanda bajo el mando de las Naciones Unidas. Estos hechos hicieron que los Estados no quisiesen ceder el control de las tropas, y prefiriesen "articular sus contribuciones al sistema de seguridad colectiva a través de organizaciones regionales como la OTAN; y, en los casos en los que han aceptado actuar bajo el mando de Naciones Unidas, a exigir ciertas adaptaciones en la dirección de las operaciones”6 . Sin embargo, tras la crisis de Kosovo se plasmó un nuevo consenso en la Declaración

\footnotetext{
${ }^{4}$ De Gucht, Karel y Keukeleire, Stephan (1991), "The European Security architecture. The role of the European Community in shaping a new European geopolitical landscape”, en Studia Diplomatica, vol. XLIV, No. 6, pp. 29 a 90

${ }^{5}$ Vid. González Alonso, Luis Norberto (2001), "La política europea de seguridad y defensa después de Niza”, Revista de Derecho Comunitario Europeo, №. 9, pp. 199207.

${ }^{6}$ González Alonso, Luis Norberto (2010), “La Unión Europea y Naciones Unidas ante la gestión internacional de crisis: Escenarios de colaboración”, Cursos de derecho internacional y relaciones internacionales de Vitoria-Gasteiz, No 1, p. 115.
} 
sobre la Defensa Europea ${ }^{7}$, tras la cumbre bilateral entre Francia y Reino Unido en Saint-Malo el 4 de diciembre de 1998.

Los objetivos de esta Declaración quedaron plasmados en el Consejo Europeo de Colonia, a través de una Declaración sobre el refuerzo de la política europea común de seguridad y defensa y a través de un informe simultáneo aprobado por los jefes de Estado y de Gobierno en el que se inicia el proceso de construcción de la Política Europea de Seguridad y Defensa ${ }^{8}$. Entre otras cosas se llegó a la conclusión de dotar a la PESD de una nueva estructura institucional en el seno del Consejo. Pero cabe destacar el propósito de generar nuevas capacidades al servicio de la Unión para la gestión internacional de crisis y la declaración de “operatividad” e inicio de las operaciones a partir del 1 de enero de 2003.

A pesar de la relevancia del marco político, el marco legal no se quedó en ningún momento atrás en estos años. A partir de Maastricht se instaura la PESC y en la reforma del Tratado de la Unión Europea, en su artículo J 11.2 se dan las primeras líneas generales en cuanto a la financiación de este sector de cooperación. Este fue el momento en el que se elaboró la distinción entre gastos operativos y gastos administrativos. Se estableció que los gastos administrativos correrían a cargo del presupuesto comunitario, mientras que los gastos operativos eran financiados por los estados miembros a no ser que el Consejo decida por unanimidad que también formaran parte del presupuesto comunitario. Es decir, se diferencian en esta política dos tipos de fuentes de financiación.

Desde entonces, estaba claro que este modelo de financiación dual, intergubernamental y comunitario iba a ser uno de los principales rasgos de la PCSD. Refleja esa tensión entre integración europea o soberanía nacional. Esta realidad crea "una asimetría jurídica en el ámbito de ejecución de la PESC" institucional y de decisión sería todavía de cooperación mientras que a la hora de la ejecución el modelo sería comunitario.

Tal y como resume Ruiz García, tras Maastricht y Ámsterdam en materia PESC y en concreto en la PCSD, existía una "falta de transparencia y de control, dificultades para determinar el coste real de las operaciones y para su reparto entre el presupuesto general de la UE y

7 El texto de esta Declaración puede consultarse en Documents d'Actualité Internationale, $\mathrm{N}^{\circ} .2$, 1999, p. 94.

${ }^{8}$ Anexo III de las Conclusiones del Consejo Europeo de Colonia (3-4 de junio de 1999)

${ }^{9}$ Liñán Nogueras, Diego Javier (1992), “La Política Exterior y de Seguridad Común de la Unión Europea”, R.I.E, vol. 19, N. 3, p. 816. 
los Estados miembros, retrasos y complicaciones en la preparación, lanzamiento y gestión de las acciones..."10.

La pregunta que estaba en el aire tras Ámsterdam era cómo se irían a articular todos estos mecanismos que por primera vez iban a dar coherencia y eficacia en la capacidad de respuesta. Fue a partir del despliegue de la operación CONCORDIA en 2003 cuando se dejó claro que se necesitaba una "solución más duradera y permanente" ${ }^{11}$ en la gestión, ya que para esta operación se creó un mecanismo ad hoc para gestionar los gastos derivados de la misma.

En este contexto y con la mirada puesta ya en futuras operaciones como la que se iba a desplegar en Bosnia, se decidió crear un nuevo mecanismo que pudiera gestionar de manera permanente este tipo de operaciones. Así es como se decidió crear el mecanismo ATHENA ${ }^{12}$.

\section{2. El mecanismo ATHENA y sus límites}

Uno de los mecanismos en los que hay que detenerse para explicar el nuevo FEAP, ya que viene a sustituirlo, es el mecanismo ATHENA. Como se ha mencionado antes, este instrumento nace de la experiencia de la operación CONCORDIA, la que fue la primera misión de la PCSD. El fondo que se utilizó para esta misión sirvió como marco de referencia para el mecanismo ATHENA. En concreto se integraron algunos elementos como: "el presupuesto para costes comunes, el Comité especial, el «administrador» y el comandante militar”" ${ }^{\text {, }}$. Lo que se pretendía con este nuevo mecanismo era gestionar los gastos comunes de manera permanente en las futuras misiones que se iban a dar.

La primera versión de este mecanismo fue la Decisión 2004/197/PESC, de 22 de diciembre. Posteriormente se ha ido

\footnotetext{
${ }^{10}$ Ruiz García, Eduardo (2017), Sistema de financiación de la política común de seguridad y defensa europea», Cuadernos de Estrategia, n 184, p. 110.

${ }^{11}$ Missirolli, Antonio (2003), "Financiación de la Política Europea de Seguridad y Defensa (PESD)”, Análisis del Real Instituto Elcano de Estudios Internacionales y Estratégicos, p. 5.

${ }^{12}$ Decisión 2004/197/PESC del Consejo, de 23 de febrero de 2004, por el que se crea un mecanismo para administrar la financiación de los costes comunes de las operaciones de la Unión Europea que tengan repercusiones en el ámbito militar o de la defensa. DO L 63, de 28.02.2004.

${ }^{13}$ Ruiz García, Eduardo (2017), “Sistema de financiación...”, pp. 126-127.
} 
actualizando tanto en su forma como en su contenido ${ }^{14}$. Las modificaciones que se han ido produciendo ha sido sobre todo la actualización de los procedimientos de gestión. En cuanto al reparto de las contribuciones, el mecanismo ATHENA se trata de un instrumento cofinanciado por los estados miembros participantes, además de los posibles acuerdos con terceros estados y organizaciones internacionales. Según el artículo 41.2 del TUE las contribuciones nacionales al presupuesto del mecanismo ATHENA, se hacen en función del producto nacional bruto de cada Estado miembro participante.

No hay que perder de vista que ATHENA gestiona y ejecuta los gastos comunes. Los demás gastos correrán a cargo de los Estados, en el que se sigue el principio "costs lie where they fall”. Alrededor de un 90 $\%$ de los gastos se corresponden a lo que aporta los Estados ${ }^{15}$. También existe la posibilidad que la financiación sea proveniente de las "agencias" de la PCSD o también pueden provenir de los "Estados miembros en colaboración con empresas privadas, otras Organizaciones Internacionales o la propia UE" ${ }^{16}$.

Es interesante comprobar como la doctrina desde los inicios del despliegue del mecanismo ATHENA, se percató de su carácter insuficiente.

En primer lugar y lo que llama más la atención es la cantidad que finalmente gestiona este mecanismo ya que, financia alrededor del $10 \%$ de los gastos extra de las misiones. Al final "los gastos recaen en los Estados participantes, lo que desincentiva la voluntad estatal de prestar efectivos para este tipo de operaciones" ${ }^{17}$.

${ }^{14}$ El estudio que se va a elaborar en este trabajo se partirá con la última actualización: Decisión (PESC) 2015/528 del Consejo, de 27 de marzo de 2015, por la que se crea un mecanismo para administrar la financiación de los costes comunes de las operaciones de la Unión Europea que tengan repercusiones en el ámbito militar o de la defensa (Athena) y por la que se deroga la Decisión 2011/871/PESC. DO L 84 de 28.3.2015.

${ }^{15}$ Vid. Fiott, Daniel (2013), “Improving CSDP Planning and Capability Development: Could there be a 'Frontex Formula'?”, European Foreign Affairs Review, 18, No 1, p. 51 y vid. Bicop, S. y Coelmont, J. (2010): "Permanent Structured Cooperation In Defence of the Obvious”, Egmont Security Policy Brief, June, 2010, p. 3.

16 Galán Pablo, Francisco (2016), "La financiación de la política exterior y de seguridad común”, tesis doctoral, UNED.

${ }^{17}$ Pérez De Las Heras, Beatriz y Churruca Muguruza, Cristina (2009), “Las capacidades militares y civiles de la Unión Europea: estado de la cuestión y propuestas de cara a la Presidencia Española 2010”, Fundación Alternativas, No: 41/2009, p.21. 
Por otro lado, se ha de tener en cuenta que este mecanismo estaba elaborado de acuerdo con el artículo 41 del Tratado de la Unión. Una de las cosas por las que es criticado este artículo es que fue redactado pensando en misiones ejecutivas, es decir aquellas estrictamente militares. Ejemplo de un tipo de misión así es la operación EUFOR, cuyos gastos correrían a cargo de los Estados miembro participantes excepto aquellos gastos comunes de los que se haría cargo ATHENA.

Sin embargo, esta distinción entre misiones ejecutivas y no ejecutivas puede plantear problemas. Es más, esta distinción afecta principalmente a la cuestión de la financiación. El ejemplo más claro de lo que se está intentando mostrar son las misiones EUTM, es decir, las misiones de entrenamiento. En una misión de entrenamiento se pueden observar tres tipos de dimensiones en el gasto; en primer lugar los gastos comunes de los que previamente se ha expuesto correrán a cargo del mecanismo ATHENA; en segundo lugar se encuentran aquellos gastos que están bajo el principio Cost lie where they fall, que normalmente estarán pagados directamente por los Estados miembros participantes y por último se encuentran aquellos gastos correspondientes al equipamiento mínimo e infraestructuras básicas para la instrucción de las Fuerzas Armadas de terceros países, en el que no se incluye armamento y munición.

El hecho es que esta última dimensión del gasto se considera de naturaleza militar y por lo tanto ha de regirse por el mecanismo ATHENA. La única alternativa para cubrir estos gastos como si fueran "costes comunes" es que el comandante acuda al artículo 15.7 del mecanismo ATHENA y solicite al Comité Especial que los considere como "costes comunes”. Para más inri, se podría dar el caso en el que el Comité Especial no se ponga de acuerdo en responder afirmativamente a tal solicitud y, por lo tanto, poner en peligro la misión de entrenamiento $^{18}$.

Y todo esto sin la más mínima intervención de las instituciones europeas. Ni la Comisión podría intervenir, como así lo hace con los fondos del FED. "El Tribunal de Cuentas únicamente podrá desplegar su capacidad para fiscalizar las cuentas respecto de aquellos gastos que

\footnotetext{
${ }^{18}$ Moreno Zamora, Juan Ignacio (2015), "Problemática de la financiación de las nuevas misiones militares de la Unión Europea”, Revista general de marina, Vol.269, p. 95.
} 
pudieran ser asumidos por el presupuesto comunitario"19 y el Parlamento Europeo tiene apenas cabida en este sistema ${ }^{20}$.

Estas limitaciones del mecanismo ATHENA tienen tres grandes consecuencias; en primer lugar, la tendencia generada por lanzar misiones con carácter exclusivamente no ejecutivo que en su mayoría son de carácter civil. En segundo lugar, la reticencia por parte de los Estados de participar en misiones de naturaleza militar debido a los costes asumidos. En tercer y último lugar que las misiones militares lanzadas por la UE son de un coste bajo, con escasos recursos y con una operatividad y capacidad muy limitada a la hora de su ejecución. En general, las misiones militares de la UE son herramientas muy limitadas ya que suelen tener un objetivo muy concreto y con escaso nivel de ambición.

\section{3. African Peace Facility y sus límites}

Otro de los mecanismos a los que viene a sustituir el nuevo mecanismo europeo de apoyo a la paz es el African Peace Facility (APF). Desde 2004 la UE ha contribuido a los esfuerzos de prevención de conflictos de la Unión Africana (UA) a través del APF. Se financia a través del Fondo Europeo de Desarrollo ${ }^{21}$ (FED) y tienen tres objetivos principales que se instrumentan a través del marco de la UA: "El apoyo financiero a operaciones de paz lideradas por esta organización, la puesta en marcha de su arquitectura de Paz y Seguridad, y el apoyo de actividades bajo el mecanismo de respuesta temprana de la UA"22. En

${ }^{19}$ Urrea Corres, Mariola (2010), “La política (común) de seguridad y defensa en el Tratado de Lisboa: La eficacia como objetivo, la flexibilidad como instrumento y la ambición propuesta”. Revista Española de Derecho Europeo, №. 33, p. 100.

${ }^{20}$ Para ver las limitaciones del Parlamento Europeo se puede ver a Urbina, Julio Jorge (2008), "Reflexiones en torno a la configuración de una Política de Seguridad y Defensa de la Unión Europea”, en Los Tratados de Roma en su cincuenta aniversario: perspectivas desde la Asociación Española de Profesores de Derecho Internacional y Relaciones Internacionales, Marcial Pons, Madrid, p. 1429.

${ }^{21}$ Es importante recalcar que el Fondo Europeo de Desarrollo nunca ha entrado en el presupuesto de la UE hasta ahora. Ahora el Fondo Europeo de Desarrollo se incluye la Rúbrica 6 del Marco Financiero Plurianual de 2021-2027. Vid. Reglamento (UE, EURATOM) 2020/2093 del Consejo de 17 de diciembre de 2020 por el que se establece el marco financiero plurianual para el período 2021-2027. DO L 433I de 22.12.2020

${ }^{22}$ Moreno Zamora, Juan Ignacio (2016), “La cuestión militar...”, pp. 443. 
resumen, se trata de un mecanismo financiero para apoyar la agenda de seguridad y paz de la UA.

Este fondo fue aprobado el 11 de diciembre de $2003^{23}$ cuya base jurídica se encuentra en el Acuerdo de Cotonú ${ }^{24}$. Existe un debate doctrinal sobre cuál fue el motivo por el que se aprobó este fondo ${ }^{25}$. A pesar de todo, fue en el contexto de la creación de la UA en 2002 cuando comienzan las primeras discusiones informales entre los representantes de la UE y la UA. Este plan se concretaría en julio de 2003 en Maputo cuando la Asamblea de la UA solicitó formalmente a la UE de crear un mecanismo de apoyo de operaciones de paz dirigidas por África ${ }^{26}$. Tiene como punto clave el principio de autonomía de decisión africana cuyas entidades beneficiarias y gestoras de estos fondos son la UA, las comunidades económicas regionales y los mecanismos regionales africanos.

Debido a las limitaciones de los Tratados, el presupuesto de la UE no puede cubrir todos los ámbitos de acción de la UE en el ámbito de la seguridad y defensa. Sin embargo, el FED, que es el principal instrumento de financiación del Acuerdo de Cotonú, no formaba parte del presupuesto de la UE y está sujeto a diferentes reglas.

Los fondos del APF vienen mayoritariamente de los sucesivos periodos del FED. Sin embargo, también existen otros medios de financiación de estos fondos. Por ejemplo, a través de compromisos anteriores del FED, dotaciones regionales del FED, aportaciones voluntarias de los Estados miembros de la UE, y en el caso del presupuesto de la UE para gastos subvencionables para el APF.

Los fondos del APF se pueden utilizar para financiar los gastos incurridos por los contingentes africanos bajo la bandera de una de las instituciones antes mencionada y que tengan que realizar una misión de

${ }^{23}$ Decisión no 3/2003 del Consejo de ministros ACP-CE, de 11 de diciembre de 2003, sobre la utilización, a efectos de la creación de un Fondo de Apoyo a la Paz para África, de recursos de la dotación para desarrollo a largo plazo del 9 FED. (DO L 345 de 31.12.2003, pp. 108-111)

${ }^{24}$ Vid. Artículo 11 del Acuerdo de Cotonú. Sitio web: http://eur-lex.europa.eu/legalcontent/EN/TXT/?uri=CELEX:02000A1215(01)-20170101

${ }^{25}$ Carbone, Maurizio (2013), “An Uneasy Nexus: Development, Security and the EU's African Peace Facility”, European foreign affairs review, Vol. 18, No Extra 1, pp. 103124.

${ }^{26}$ Assembly of the African Union, Decisions and Declarations, Assembly/AU/Dec.21 II, Decision on the Establishment by the European Union of a Peace Support Operation Facility for the African Union, Maputo, (10-12 Jul. 2003). 
paz y seguridad bajo el mandato de la UA. Entre los gastos que cubre este mecanismo, por ejemplo, se encuentra el coste de transporte de las tropas, los gastos de manutención de los soldados o el desarrollo de las capacidades. Entre los gastos que no se incluyen dentro del APF son aquellos como las municiones, las armas y el equipo militar, repuestos de equipos militares, salarios o entrenamiento militar para soldados ${ }^{27}$. Como se verá más adelante, este es uno de los objetivos que trata de atajar el nuevo mecanismo y que todavía está pendiente de sus efectos.

Más del $90 \%$ de los fondos del APF se han gastado en operaciones de apoyo a la paz. Este instrumento ha contribuido a la financiación de dieciséis misiones dirigidas en África como la Misión de la Unión en Somalia (AMISOM), cuya mayor parte ha cubierto los gastos de los salarios de las tropas o MISCA en la República Centroafricana (2013-2014) o la MNJTF para la lucha contra el Boko Haram en la cuenca del lago Chad $^{28}$.

Sin embargo, llama la atención que la mayoría de los fondos han ido destinados a una operación en concreto. Desde 2007 hasta 2019, más de 1.94 mil millones de euros han ido destinado a la operación de AMISOM $^{29}$.

No hay que olvidar que además del apoyo operativo, también se fomenta el diálogo sobre la paz y la seguridad, así como la puesta en funcionamiento de la Arquitectura Africana de Paz y Seguridad (APSA). Este apoyo institucional que brinda la UE fue tiene como objetivo complementar la financiación de las operaciones con miras a reforzar la capacidad de la UA y los demás organismos regionales asociados para gestionar el despliegue, el mando y el control de las operaciones de paz.

Entre otras cosas, los fondos destinados al apoyo institucional cubren la formación y los salarios de la Comisión de la UA, la

\footnotetext{
27 “African Peace Facility, Annual Report 2019”, Directorate General for International Cooperation and Development (DG DEVCO), julio, 2020.

${ }^{28}$ Boutillier, Clément (2017), “Development cooperation and crisis management”. Recasting EU civilian crisis management, EU Institute for Security Studies, n⿳⺈ 31, pp. 46.

${ }^{29}$ La mayor parte de la dotación del APF entre 2004 y 2019 se asignó a las operaciones de paz con un 93\% (2.681,2 millones de euros). Luego la arquitectura de las capacidades ha supuesto alrededor del $6 \%$ (con 171.8 millones de euros) y finalmente el mecanismo de Alerta Temprana supone un 1\% (28 millones de euros). Para ver con más detalle vid. “African Peace Facility, Annual Report 2019”... pp. 9 y 10.
} 
infraestructura y el apoyo técnico de la $\mathrm{UA}^{30}$. Sin embargo, la cantidad de dinero que va dirigida al apoyo institucional es bastante reducido ${ }^{31}$.

Más reducida es la cantidad dirigida al Mecanismo de Alerta Temprana, que trata de responder a las solicitudes de financiación de la UA en tan solo diez días hábiles. Esto se suele utilizar para preparar las primeras fases de una operación que se quiere llevar a cabo. Sobre todo, se llevan a cabo medidas preventivas como el de la mediación, la diplomacia o el despliegue de observadores de derechos humanos. Se han llevado a cabo más de 40 iniciativas y sin lugar a duda se trata de uno de los programas más exitosos del APF a pesar de recibir únicamente 28 millones de euros desde su creación en 2009 hasta $2018^{32}$.

Es mucho más claro el caso de los límites que ofrece el APF ya que se enmarca en los Fondos Europeos de Desarrollo. Una de las características del FED es que se trata de un instrumento de Ayuda Oficial al Desarrollo (AOD), y por lo tanto está sujeto a las normas de la OCDE sobre los gastos en ayudas que restringen los pagos para actividades militares. ${ }^{33}$ Si bien es cierto que en 2016 se flexibilizaron estas normas, estas continúan estipulando que todas aquellas ayudas en seguridad y en materia militar deben tener unos fines de desarrollo ${ }^{34}$. Además, estos fondos tienen como fundamento jurídico el Acuerdo de Cotonú, que en su artículo 11 sobre las políticas en favor de la paz, prevención y resolución de los conflictos, en su disposición cuarta nos habla que:

“[...] Se dedicará un esfuerzo especial a garantizar que los recursos financieros de la cooperación se utilicen de acuerdo con los principios y los objetivos de la asociación y a impedir el desvío de los fondos para fines bélicos”

\footnotetext{
30 "How to Spend It: New EU Funding for African Peace and Security”, Crisis Group Africa Report, $\mathrm{N}^{\circ} 297,14$ de enero de 2021, pp. 3.

31 Para profundizar más en este aspecto del APF, vid. Pirozzi, Nicoletta (2009), "EU support to African Security architecture: funding and training components", Occasional Paper, Instituto de Estudios de Seguridad de la UE, N N $^{\circ}$.

${ }^{32}$ Vid. “African Peace Facility, Annual Report 2019”... pp. 5

${ }^{33}$ Furness, Marck y Bergmann, Julian (2018), “A European peace facility could make a pragmatic contribution to peacebuilding around the world”, Deutsches Institut für Entwicklungspolitik (DIE), Briefing Paper, № 6, Bonn.

${ }^{34}$ Concord (2018), "Security aid: Fostering development or serving European donors' national interest?”, European NGO confederation for relief and development, AidWatch, Bruselas.
} 
Es decir, los recursos que vienen de este fondo no podían sobrepasar ciertos límites por el mismo hecho de pertenecer a un fondo de desarrollo. Por ejemplo, no pueden financiarse a través de estos la munición, armas o equipos de repuesto. También está prohibido los salarios de los militares o los soldados que van a una operación de entrenamiento $^{35}$.

Otra limitación que se daba desde los comienzos es el problema institucional generado, ya que, desde Maastricht, la política de seguridad era competencia del Consejo, pero la utilización y gestión de este fondo estaba a cargo de la Comisión. Por lo tanto, esto ha sido óbice para extender la competencia de la Comisión en gestión de prevención de conflictos y en la reconstrucción tras los conflictos. Lo que se plantea de fondo es lo que se pregunta el Comisionario de Desarrollo, “¿Se trataba de política exterior o era cooperación al desarrollo?” ${ }^{36}$. Cualquiera de las soluciones propuestas se consideraba que alteraba el delicado equilibrio entre las dos instituciones europeas ya que los funcionarios de la Comisión temían que el Consejo empezara a entrometerse en cuestiones sobre desarrollo y dentro del Consejo los responsables políticos temían que la Comisión Europea buscara una mayor implicación en la política de seguridad de la UE.

En definitiva, no se tienen los medios ni las capacidades militares que exigirían a la UE una implicación más directa en las misiones llevadas a cabo por la UA. Esto se debe entre otras cosas el poco margen de iniciativa que tiene la UE en liderar estas misiones y el límite que tiene a través de este APF al tratarse de un fondo de desarrollo.

\section{EL NUEVO FONDO EUROPEO DE APOYO A LA PAZ}

Durante una comparecencia del 13 de diciembre de 2017, la Alta Representante anunció un nuevo fondo extrapresupuestario para financiar las misiones militares de la Unión ${ }^{37}$. Esta propuesta se presentó

\footnotetext{
35 Santopinto, Federico y Marèchal, Julien (2021), "EU military assistance under the new European Peace Facility”. Konrad Adenauer stifung.

${ }^{36}$ Nielson, Poul (2012), "EU Aid: What Works and Why”, UNU-Wider Working Paper 76.

37 Mogherini, Federica (2017), Comparecencia de la Alta Representante y Vicepresidenta de la Comisión en el evento: "Building on vision, forward to action: delivering on EU security and defence”. Bruselas, 13 diciembre de 2017.
} 
formalmente en junio de 2018 por parte de la Alta Representante ${ }^{38}$. Tras un largo periodo de discusiones y debates, el 22 de marzo se aprobó el nuevo mecanismo europeo por la que se crea un Fondo Europeo de Apoyo a la Paz y se deroga la Decisión (PESC) 2015/528.

Vistos los diferentes mecanismos que se han utilizado para apoyar financieramente a las misiones en el ámbito militar y de defensa y las diferentes limitaciones que poseían es conveniente analizar en primer lugar el nuevo FEAP de una manera descriptiva para luego analizar el fondo del asunto.

A modo de introducción, llama la atención que este nuevo mecanismo se asemeje en gran medida al mecanismo ATHENA que es al que viene a sustituir. De ahí que este trabajo se centre más en las diferencias de ambos mecanismos.

\section{1. Aspectos generales}

Una de las novedades que introduce este nuevo mecanismo está en el ámbito de aplicación. En el artículo 1 de la Decisión, enuncia que el Fondo además de utilizarse para los costes comunes de las operaciones de la Unión realizadas al amparo del artículo 42, apartado 4, y del artículo 43, apartado 2, del TUE que tengan repercusiones en el ámbito militar o de la defensa también se utilizará para financiar las medidas de asistencia. Estas medidas de asistencias a las que se refiere son en realidad las acciones para reforzar las capacidades de terceros Estados y organizaciones regionales e internaciones en relación con asuntos militares y de defensa y el apoyo a los aspectos militares de las operaciones de apoyo a la paz dirigidas por una organización regional o internacional o por terceros Estados. Además, en el artículo 4 añade que las medidas de asistencia podrán consistir en apoyo financiero, técnico o material. Además, dicha acción podrá adoptar la forma de una medida especifica o de un programa general de apoyo con un enfoque geográfico o temático determinado ${ }^{39}$. Estas medidas de asistencia a las que se refiere el nuevo Fondo son aquellas que corresponderían al APF.

\footnotetext{
38 "Proposal of the High Representative of the Union for Foreign Affairs and Security Policy, with the support of the Commission, to the Council of 13/06/2018 for a Council Decision establishing a European Peace Facility”, Servicio de Acción Exterior Europeo, 13 de junio, Bruselas.

${ }^{39}$ Artículo 4 Decisión (PESC) 2021/509 del Consejo.
} 
Además, en el considerando 18 se recuerda que el ámbito de aplicación de este instrumento tendrá un alcance geográfico mundial. Este recordatorio sirve para ampliar el espectro, ya que el APF se limitaba exclusivamente al territorio africano. Esto no quiere decir que se interrumpan los compromisos y apoyos prestados a África. Sin embargo, si se pondrá énfasis sobre todo a aquellos países y regiones que presentas las amenazas más urgentes y críticas para la seguridad de la Unión.

Como se puede comprobar en el artículo 7 del FEAP, es el Consejo quien ahora toma todas las decisiones, incluidas las medidas de asistencia. Este es otro gran cambio, ya que con el APF la decisión final recaía en la Comisión ya que se trataba de un fondo de desarrollo. Ahora, se encuadra sin duda alguna en una competencia relativa a la seguridad y defensa y por lo tanto es el Consejo quien tendrá la última palabra.

En cuanto la organización, la estructura orgánica es prácticamente idéntica al mecanismo ATHENA. Si bien, el hecho de que se haya incluido las medidas de asistencia en el ámbito de aplicación hace que se incluya un administrador de las medidas de asistencia. Por lo tanto, aparte de haber un Comité del Fondo, un administrador de las operaciones y un contable de las operaciones, también se crea la figura del administrador y contable para las medidas de asistencia.

También se ha profundizado en las competencias de la estructura organizativa del nuevo Fondo. Por ejemplo, se ha cubierto la laguna con relación al Comité en el caso de no llegar a un acuerdo sobre un punto concreto. Ahora si se da el caso, el Comité podrá decidir remitir el asunto al Consejo para que adopte una Decisión ${ }^{40}$.

Con la implementación del Comité Político y del Alto Representante, la UE trata de dar una solución a dos modelos de gobernanza que se entremezclan. Por un lado, el intergubernamental del mecanismo ATHENA y luego el APF que contiene elementos de la gobernanza de la política de desarrollo de la UE. Si bien es cierto que la orientación de este nuevo Fondo es exclusivamente militar y por lo tanto requerirá la decisión unánime del Consejo para la toma de decisiones, también es cierto que, en determinados casos, el Consejo necesitará la aprobación del Alto Representante o del Comité Político y Seguridad.

Por ejemplo, el apoyo de cualquier acción comprendida en un programa general será a iniciativa del beneficiario y requerirá el examen

${ }^{40}$ Vid art 11.19 Decisión (PESC) 2021/509 del Consejo 
y la aprobación previas del Comité Político y de Seguridad ${ }^{41}$. Sin embargo, las medidas que se tomen estarán bajo el control de los Estados miembros. Bastaría la oposición de un solo Estado miembro en la toma de una decisión para impedir que se aplique ${ }^{42}$. Ya sea o en el Comité Político y de Seguridad o bien en el Consejo.

\section{2. Gastos comunes y contribuciones de los Estados miembros.}

Una de las diferencias más notables con el mecanismo ATHENA es que el nuevo FEAP proporcionaría un fondo permanente no sólo para hacer más predecible y flexible la financiación de las operaciones militares de la PCSD, sino también para ampliar el alcance de los gastos comunes $^{43}$.

Los costes comunes se siguen agrupando como el anterior mecanismo si bien se amplía por ejemplo el concepto en los costes comunes relativos a la fase activa de las operaciones de la Unión, que correrán a cargo del fondo en los que se incluye los costes relativos al despliegue de los Battlegroups ${ }^{44}$.

Igualmente, la propuesta del nuevo Fondo tiene por objeto aumentar el alcance de los costes. Se estima que el alcance con el mecanismo ATHENA se sitúa entre el 5\% y el 15\% de los costes totales de la misión, dependiendo del tipo de misión. La propuesta del nuevo Fondo elevaría este porcentaje hasta un $35 \%$ y $45 \%$ como máximo ${ }^{4546}$. Este fondo se ha visto reducido en comparación con la propuesta de la Comisión de 2018 que preveía desembolsar alrededor de 9.200 millones de euros ${ }^{47}$. Aunque se trate de un fondo extrapresupuestario, funcionará de forma paralela al marco financiero plurianual 2021-2027. Esto permite a los Estados

\footnotetext{
${ }^{41}$ Vid art 59.7 Decisión (PESC) 2021/509 del Consejo

${ }^{42}$ Vid. Deneckere, Matthias (2019), "The uncharted path towards a European Peace Facility”, EDCPM, Discussion paper $\mathrm{N}^{\circ} .248$.

${ }^{43}$ Ibidem.

${ }^{44}$ Vid. Anexo IV, Parte A, apartado 3 Decisión (PESC) 2021/509 del Consejo

45 "Proposal of the High Representative..."

${ }^{46}$ Vid Anexo I Decisión (PESC) 2021/509 del Consejo. Reparte la financiación en los diferentes periodos. En 2021 se repartirán 420 millones de EUR; en 2022 - 540 millones de EUR; en 2023 - 720 millones de EUR; en 2024 - 900 millones de EUR; en 2025 980 millones de EUR; en 2026 - 1.000 millones de EUR y en 2027 - 1.132 millones de EUR.

${ }^{47}$ Vid. Hauck, Volker (2020), “The latest on the European Peace Facility and what's in it for the African Union” EDCPM, Briefing note $\mathrm{N}^{\circ} .120$.
} 
miembros calcular la cantidad que se van a ir gastando. Lo más interesante de todo esto es que al vincular el nuevo Fondo con el MFP, los Estados miembros pueden decidir las asignaciones financieras del FEAP como parte de un debate más amplio sobre cuánto desean gastar en la acción exterior en general, y qué parte de las actividades en el ámbito militar o de la defensa deben ocupar dentro de este marco.

\section{3. Medidas de control y acompañamiento.}

La cuestión más controvertida que ha generado este nuevo mecanismo es el hecho de que a partir de ahora la Unión podrá suministrar armas letales a terceros países. Estos nuevos suministros que se ofrecen podrían acabar en las manos equivocadas y ser utilizadas para cometer atrocidades, lo que, a su vez, podría dar lugar a intentos de negar pruebas o eludir la responsabilidad.

Esto no sucedía antes ya que el precedente del APF limitaba estas cuestiones al formar parte del fondo de desarrollo. Por ello, el Consejo ha establecido un marco integrado de mecanismos de cumplimiento y control a los que deberá adherirse el beneficiario ${ }^{48}$ que reciba el equipo militar. Por ejemplo, en el artículo 64 se disponen los casos en los que el Comité Político y de Seguridad podrá decidir suspender total o parcialmente la ejecución de una medida de asistencia, a petición de un Estado miembro o del Alto Representante. Entre los casos se encuentra aquel en el que el beneficiario incurra en incumplimiento de sus obligaciones en virtud del derecho internacional o si incumple los compromisos asumidos en virtud del artículo 62 de la misma Decisión.

Del mismo modo, el artículo 9 del mecanismo prevé que el Consejo y el Comité Político y de Seguridad revisar periódicamente un método para el análisis de riesgos y salvaguardias respecto a las medidas de asistencia que se financien con cargo al fondo ${ }^{49}$. Este método comprenderá los posibles componentes de mitigación y acompañamiento, disposiciones de seguimiento y la evaluación, y controles y salvaguardias ${ }^{50}$. Pues bien, este método se ha plasmado en un documento denominado Marco Metodológico Integrado. Se rige por una serie de

\footnotetext{
48 Siempre que se hable de beneficiario se está refiriendo a un tercer Estado o una organización internacional a quien presta apoyo una medida de asistencia.

${ }^{49}$ Vid art 9.3 Decisión (PESC) 2021/509 del Consejo

${ }^{50}$ Vid art 9.5 Decisión (PESC) 2021/509 del Consejo
} 
principios como el de cumplimiento, de protección de la UE y de las poblaciones locales, de proporcionalidad o de control posterior a la entrega. Establece un proceso que se divide en dos fases; una ex ante y otro ex post y establece una serie de medidas en caso de incumplimiento.

\section{UNA MIRADA PROSPECTIVA DEL NUEVO FONDO}

\subsection{El nuevo Fondo en el contexto de la PCSD}

Visto a grandes rasgos las novedades que ofrece este nuevo mecanismo, resulta interesante encuadrarlo en un marco más amplio para poder entender su sentido último. La realidad es que desde 2016 se ha llevado a cabo un impulso notorio en el sistema de la PCSD a nivel institucional. Elementos como la Cooperación Estructurada Permanente (PESCO), la revisión anual coordinada de defensa (CARD), el nuevo Fondo Europeo de Defensa (EDF), que se centra sobre todo en la industria armamentística, o la Capacidad de Planificación y Conducta Militar (en inglés Military Planning and Conduct Capability o MPCC), además del Fondo Europeo de Apoyo a la Paz, muestran un avance en el sistema institucional que se debe a diferentes circunstancias. Entre otras, la evolución del panorama geoestratégico, tanto por la aparición de nuevos actores como China o el incremento de las tensiones con los países vecinos como Rusia, hace que la UE tenga que tratar de poner más medios para ganar en capacidades y así ganar mayor autonomía ${ }^{51}$. Es por eso por lo que se crea entre otros el FEAP ya que podrá dotar de equipo militar, incluyendo armas y municiones a los socios y así conseguir que la UE pueda ofrecer un paquete integrado de apoyo para el desarrollo de las capacidades. Es un paso más en esta dirección ${ }^{52}$.

Especial atención merece el estudio de la nueva configuración institucional y de sus repercusiones. Ahora el Consejo será el responsable de tomar las decisiones más importantes respecto a las misiones militares pasando la Comisión a un segundo plano. Se deja a un lado el sistema

\footnotetext{
${ }^{51}$ El distanciamiento de Estados Unidos estos últimos años venía siendo una de las causas del ritmo acelerado de desarrollo de la UE en materia de defensa. Sin embargo, el pasado 15 de junio de 2021 se firmó un documento entre la UE y Estados Unidos definiendo los objetivos a seguir. Entre ellos destaca el $n^{0} 36$ en el que se refuerzan los lazos en materia de seguridad de la UE y la OTAN. Vid. EU-US Summit Statement. «Towards a renewed Transatlantic partnership», 15 de junio de 2021, Bruselas.

${ }^{52}$ Hagström Frisell, Eva y Sjökvist, Emma (2021), “To Train and Equip Partner Nations - Implications of the European Peace Facility”, FOI: International Military Missions, Swedish Defence Research Agency.
} 
que imponía el APF en el que el poder de iniciativa recaía sobre la Comisión y los programas de acción necesitaban una mayoría cualificada del COREPER. Al dar a los Estados miembros tanto el derecho a presentar propuestas como su capacidad de veto en la toma de decisiones, los países tienen ahora más capacidad para imponer sus prioridades en la agenda. El hecho de que se haya fijado el nuevo Fondo de forma paralela al Marco financiero plurianual de 2021-2027 da a entender que el voto en el Consejo se utilizará como moneda de cambio en cuestiones presupuestarias y estratégicas. Además, se ha incluido en el FEAP nuevas disposiciones relativas a la actuación del Consejo en el caso de que haya que tomar medidas de $\operatorname{urgencia}^{53}$. Es decir, en teoría, gracias a esta reunificación institucional que va a encarnar el Consejo habrá mayor eficacia y rapidez a la hora de responder ante una necesidad. Sin embargo, también es verdad que esto puede plantear alguna disyuntiva ya que los Estados miembros pueden actuar con una visión cortoplacista y acabar los recursos compartidos sin una política estratégica a largo plazo. De ahí la necesidad de implementar programas de acción plurianuales que definan los objetivos a alcanzar durante un periodo de tiempo ${ }^{54}$.

En relación con el poder del Consejo, la doctrina cuestiona la nueva capacidad otorgada de poder suministrar armas y municiones a terceros estados o a entidades no estatales y su capacidad de gestión. De hecho, una de las preguntas que está en el aire es si la UE será capaz de promover las reformas necesarias para mitigar los riesgos que implica el aumento de su apoyo en el equipamiento militar ${ }^{55}$. Las medidas de control y acompañamiento que se han visto más arriba se refieren únicamente a la suspensión de la financiación en el caso de que no se cumplan los objetivos establecidos y como máximo, el Comité Político y de Seguridad podrá recomendar al Consejo la rescisión de una mediada de asistencia. Sin embargo, esto puede resultar insuficiente.

Todavía es pronto para saber si esto será realmente una desventaja notable, pero la poca transparencia que hay en estas situaciones y la dificultad que hay de aplicar estos controles en la práctica, hacen que haya numerosas voces críticas al respecto. Sobre todo, ven poco coherente la estructura del nuevo Fondo ya que como afirma el

\footnotetext{
${ }^{53}$ Vid. arts. 28 y 58 Decisión (PESC) 2021/509 del Consejo.

${ }^{54}$ Vid Deneckere, Matthias (2019), «The uncharted path towards...», pp. 13.

${ }^{55}$ Hagström Frisell, Eva y Sjökvist, Emma (2021),: «To Train and Equip Partner Nations...», pp. 8.
} 
considerando 15 del FEAP, ha de haber coherencia y complementariedad entre este mecanismo y los instrumentos de financiación exterior de la Unión, y en particular con el Instrumento de Vecindad, Desarrollo y Cooperación Internacional y otras políticas de la Unión, cuando corresponda. Es el Consejo quien se encargará de velar por la coherencia a todos niveles, y el Comité Político y de Seguridad quien debe proporcionar orientación estratégica para las operaciones militares y las medidas de asistencia de la Unión que se financiarán con cargo al Fondo, en particular para garantizar la coherencia y la complementariedad de la PESC, incluida la PCSD ${ }^{56}$.

\subsection{Un nuevo mecanismo para África}

Por último, es probable que la capacidad de ayuda a terceros en términos de capacidades militares se reduzca. Hay que recordar que este mecanismo hereda todas las misiones vigentes del APF. Por lo tanto, teniendo en cuenta los compromisos actuales para el apoyo de las operaciones militares africanas, y AMISOM en particular, el presupuesto de 5.000 millones de euros del nuevo Fondo deja mucho menos espacio para la adquisición de equipo militar de lo que habían concebido algunos al principio ${ }^{57}$. Sin embargo, esto no garantiza que las misiones que ya está operativas en África a través de la UA estén totalmente aseguradas el pago. De hecho, uno de los problemas a los que se presenta la UA es la más que probable reducción de la asignación que suministra la UE para estas misiones. En particular, preocupa la misión de AMISOM ya que en 2016 hubo un precedente en el que la UE tuvo que recortar el $20 \%$ de lo asignado. Esto generó grandes tensiones ya que la UE es el máximo contribuidor en esta misión ${ }^{58}$.

Una idea que late de fondo es que, para los países africanos podría suponer un peligro que el nuevo Fondo pase a ser un instrumento global en lugar de estar dedicado específicamente a la seguridad africana. Sin embargo, el hecho de que sea un instrumento global con demandas contrapuestas debería incentivar a los gobiernos africanos a un

\footnotetext{
${ }^{56}$ Vid. Considerando No 15 Decisión (PESC) 2021/509 del Consejo.

${ }^{57}$ Hauck, Volker (2020), “The latest on the European Peace Facility...”, pp. 8.

58 Vid. S Mahmood, Omar y Christian Ani, Ndubuisi (2017), "Impact of EU funding dynamics on AMISOM”, Institute for Security Studies.
} 
compromiso más firme y a una contribución más generosa con la Unión Africana ${ }^{59}$.

Por el momento se ha aprobado una Decisión del Consejo en octubre de 2020 para asegurar la continuidad de la financiación de las diferentes actividades del APF hasta el 30 de junio de 2021 o hasta la expiración del Acuerdo de Cotonú. La cantidad asignada para este periodo es de 113 millones de euros ${ }^{60}$.

Las grandes ventajas que presenta este nuevo Fondo se traducen en una mayor flexibilidad por parte de la Unión Europea. A partir de ahora la UE podrá financiar directamente las operaciones de apoyo a la paz y a las coaliciones militares incluso cuando no estén operando bajo el mandato del Consejo de Seguridad y Paz de la Unión Africana. Además, la UE podrá financiar con este nuevo Fondo el apoyo bilateral con los ejércitos africanos. Esto se ve como una oportunidad para profundizar en la cooperación con los Estados africanos como es el caso de Mali o Somalia. Con estos países la intención es aumentar el apoyo bilateral en las misiones de entrenamiento o en el desarrollo de las capacidades militares ${ }^{61}$.

\section{CONCLUSIONES}

En definitiva, este nuevo FEAP es algo más que un reajuste de los instrumentos que había entonces, sino que lleva la política de paz y seguridad de la UE un paso más allá. Se le está dando a la UE de una nueva herramienta que le permite desplegar las operaciones con mayor flexibilidad y rapidez. Además, también se le dota de mayores opciones de asistencia a los socios de desarrollo de la capacidad militar. El FEAP se ha aprobado justo en un momento de incertidumbre geopolítica y de ahí que este instrumento sirva para reforzar las escasas capacidades militares de la UE.

\footnotetext{
${ }^{59}$ Furness, Mark y Bergmann, Julian (2018), “A European peace facility could...”, pp. 4.

60 Decisión (UE) 2020/1422 del Consejo de 5 de octubre de 2020 relativa a la asignación de fondos liberados de proyectos con cargo al 10.o Fondo Europeo de Desarrollo con el fin de reaprovisionar el Fondo de Apoyo a la Paz para África. DO L 329 de 9.10.2020.

61 “How to Spend It: New EU Funding for...”, pp. 2.
} 
Sin embargo, existen algunos dilemas que se tendrá que ir viendo poco a poco como se van desarrollando. Todavía es pronto, y aunque este fondo tiene como objetivo último consolidar la paz y prevenir conflictos, se va a aumentar el riesgo de que las posibles ayudas en capacidades militares a terceros caigan sobre manos indebidas. Por lo tanto, es necesario garantizar las salvaguardias y los sistemas de control para evitar que la ayuda militar refuerce, en lugar de aliviar la dinámica del conflicto. El nuevo mecanismo aprueba una serie de procedimientos de control y parece preocupado por este asunto. Pero existe la duda de hasta qué punto estas medidas serán efectivas ya que en estas situaciones suele ser muy complejo el hecho de tener que controlar estos aspectos. El Consejo acaba de confirmar un documento no público en el que se establecen el método a la hora de implementar esas medidas de control. Está por ver cómo se va a ejecutar y la efectividad de dicho documento. Además, tampoco podrá controlar el Parlamento Europeo las medidas que se vayan ejecutando ya que se trata de un mecanismo extrapresupuestario. Únicamente será informado de la aplicación del nuevo Fondo.

Asimismo, el impacto final del FEAP como instrumento financiero difiere con lo inicialmente propuesto por los Estados miembros de la UE. En vista de la pandemia del COVID-19, y otras prioridades identificadas durante las negociaciones para el presupuesto de julio de 2020 hizo que se debilitaran las propuestas en seguridad y defensa. De hecho, el presupuesto para este Fondo ha sufrido un recorte del $46 \%$ en comparación con lo que había propuesto inicialmente la Alta Representante en 2018. Además, en comparación con las líneas presupuestarias del Marco Financiero Plurianual 2021-2027, y en particular en comparación con la rúbrica 6 que cubre los aspectos de vecindad y el resto del mundo, el recorte ha sido mucho más severo. Bien es verdad que este nuevo Fondo, sin ser un mecanismo presupuestario, se ha vinculado al MFP de 2021-2027 para que se tenga en cuenta a la hora de los debates entre los países miembros.

En resumen, el FEAP supone un pequeño avance en el conjunto del desarrollo de la PCSD, sin embargo, va a ser uno de los mecanismos en el que más se va a visibilizar hasta qué punto se comprometen los Estados miembros en materia de Seguridad y Defensa. De momento, esto nos lleva a pensar la poca relevancia estratégica del FEAP, tanto en términos de dinero como la falta de liderazgo en esta materia. 


\section{BIBLIOGRAFÍA}

Besch, Sophia (2020), “EU’s Institutional Framework regarding Defence Matters”, Policy Department, Directorate-General for External Policies, Parlamento Europeo, Bruselas.

Biscop, Sven y Coelmont, Jo (2010), “Permanent Structured Cooperation in Defence of the Obvious”, Egmont Security Policy Brief.

Boutillier, Clément (2017), "Development cooperation and crisis management”. Recasting EU civilian crisis management, EU Institute for Security Studies, № 31.

Concord (2018), "Security aid: Fostering development or serving European donors' national interest?”, European NGO confederation for relief and development, AidWatch, Bruselas.

Carbone, Maurizio (2013), “An Uneasy Nexus: Development, Security and the EU's African Peace Facility", European foreign affairs review, Vol. 18, $\mathrm{N}^{\circ}$ Extra 1.

De Gucht, Karel y Keukeleire, Stephan (1991), “The European Security architecture. The role of the European Community in shaping a new European geopolitical landscape”, en Studia Diplomatica, vol. XLIV, $\mathrm{N}^{\circ} .6$.

Deneckere, Matthias (2019), "The uncharted path towards a European Peace Facility”, EDCPM, Discussion paper No 248.

Elías Boada, Joan (2001), "El Consejo y el Parlamento Europeo ante la financiación de la PESC”, Working Paper, No. 15, Observatorio de 
Política Exterior Europea, IUEE, Universidad Autónoma de Barcelona.

Fiott, Daniel (2013), "Improving CSDP Planning and Capability Development: Could there be a 'Frontex Formula'?”, European Foreign Affairs Review, 18, No 1.

Furness, Mark y Bergmann, Julian (2018), “A European peace facility could make a pragmatic contribution to peacebuilding around the world”, Deutsches Institut für Entwicklungspolitik (DIE), Briefing Paper, Nº 6, Bonn.

Galán Pablo, Francisco (2016), “La financiación de la política exterior y de seguridad común”, tesis doctoral, UNED.

González Alonso, Luis Norberto (2001), "La política europea de seguridad y defensa después de Niza”, Revista de Derecho Comunitario Europeo, №. 9.

González Alonso, Luis Norberto (2010), “La Unión Europea y Naciones Unidas ante la gestión internacional de crisis: Escenarios de colaboración”, Cursos de derecho internacional y relaciones internacionales de Vitoria-Gasteiz, № 1.

Hagström Frisell, Eva y Sjökvist, Emma (2021), “To Train and Equip Partner Nations - Implications of the European Peace Facility”, FOI: International Military Missions, Swedish Defence Research Agency.

Hauck, Volker (2020), "The latest on the European Peace Facility and what's in it for the African Union” EDCPM, Briefing note $\mathrm{N}^{\circ} .120$.

Liñán Nogueras, Diego Javier (1992), "La Política Exterior y de Seguridad Común de la Unión Europea”, R.I.E, vol. 19, № 3.

Missiroli, Antonio (2003), "Financiación de la Política Europea de Seguridad y Defensa (PESD)”, Análisis del Real Instituto Elcano de Estudios Internacionales y Estratégicos 
Moreno Zamora, Juan Ignacio (2015), “Problemática de la financiación de las nuevas misiones militares de la Unión Europea”, Revista general de marina, Vol.269

Moreno Zamora, Juan Ignacio (2016), “La cuestión militar en la Unión Europea”, Instituto Español de Estudios Estratégicos, № 3

Nielson, Poul (2012), "EU Aid: What Works and Why”, UNU-Wider Working Paper, N$^{\circ} .76$.

Pérez De Las Heras, Beatriz y Churruca Muguruza, Cristina (2009), “Las capacidades militares y civiles de la Unión Europea: estado de la cuestión y propuestas de cara a la Presidencia Española 2010”, Fundación Alternativas, No: 41/2009

Pirozzi, Nicoletta (2009), "EU support to African Security architecture: funding and training components", Occasional Paper, Instituto de Estudios de Seguridad de la UE, Nº 76.

Robles Carrillo, Margarita (1995), “La financiación de la PESC”, Revista de Instituciones Europeas, vol. 22, Nº.1.

Ruiz García, Eduardo (2017), “Sistema de financiación de la política común de seguridad y defensa europea”, Cuadernos de Estrategia, $\mathrm{n}^{\circ} 184$.

Santopinto, Federico y Marèchal, Julien (2021), "EU military assistance under the new European Peace Facility”. Konrad Adenauer stifung.

S Mahmood, Omar y Christian Ani, Ndubuisi (2017), "Impact of EU funding dynamics on AMISOM”, Institute for Security Studies.

Urbina, Julio Jorge (2008), "Reflexiones en torno a la configuración de una Política de Seguridad y Defensa de la Unión Europea”, en Los Tratados de Roma en su cincuenta aniversario: perspectivas desde la Asociación Española de Profesores de Derecho Internacional y Relaciones Internacionales, Marcial Pons, Madrid. 
Urrea Corres, Mariola (2010), "La política (común) de seguridad y defensa en el Tratado de Lisboa: La eficacia como objetivo, la flexibilidad como instrumento y la ambición propuesta”. Revista Española de Derecho Europeo, N ${ }^{\circ} .33$. 